\title{
Effect of thiazolidinediones - PPARY agonists on hormones secretion by cells of human adrenocortical cancer in vitro
}

\author{
Julita Fuss-Chmielewska ${ }^{1}$, Hanna Eawnicka ${ }^{2}$, Jacek Świętosławski ${ }^{1}$, \\ Karolina Beda-Maluga ${ }^{1}$, Katarzyna Winczyk ${ }^{1}$
}

${ }^{1}$ Department of Neuroendocrinology, Chair of Laboratory Medicine, Medical University of Łódź, Poland ${ }^{2}$ Department of Immunoendocrinology, Chair of Endocrinology, Medical University of Łódź, Poland

\section{INTRODUCTION}

Adrenocortical carcinoma (ACC) is characterized by the high malignancy of the lesion, poor prognosis and high mortality rate. The surgical treatment and available chemotherapy have generally low efficacy so the search for new therapeutic options is still necessary. Some research indicated that thiazolidinediones (TZDs) - synthetic ligands of peroxisome proliferators-activated receptor $\gamma$ (PPARY) have oncostatic action on neoplasm, including endocrine tumours [1-3]. Moreover, previous investigations have shown the inhibitory effect of TZDs on the growth of human adrenocortical cancer [4-6]. Therefore, in the present study, we decided to examine whether TZDs influence the secretion of adrenocortical hormones.

\section{MATERIAL AND Methods}

The experiment was conducted on human adrenocortical carcinoma line H295R. The cancer cells were treated with two TZDs rosiglitazone (ROS) or pioglitazone (PIO) at the concentrations $10^{-8}-10^{-5} \mathrm{M}$ for three days. In the culture medium, we measured the levels of the following hormones: aldosterone, cortisol, dehydroepiandrosterone sulfate (DHEAs), androstendione and 17-hydroxyprogesterone by using chemiluminescence immunoassay and radioimmunoassay.

\section{RESULTS}

Both TZDs at most of the concentrations have increased DHEAs level. Besides, PIO stimulated cortisol secretion by cancer cells at all tested concentrations and ROS increased cortisol level only at the highest concentration. However, TZDs did not change significantly the levels of other adrenal hormones.

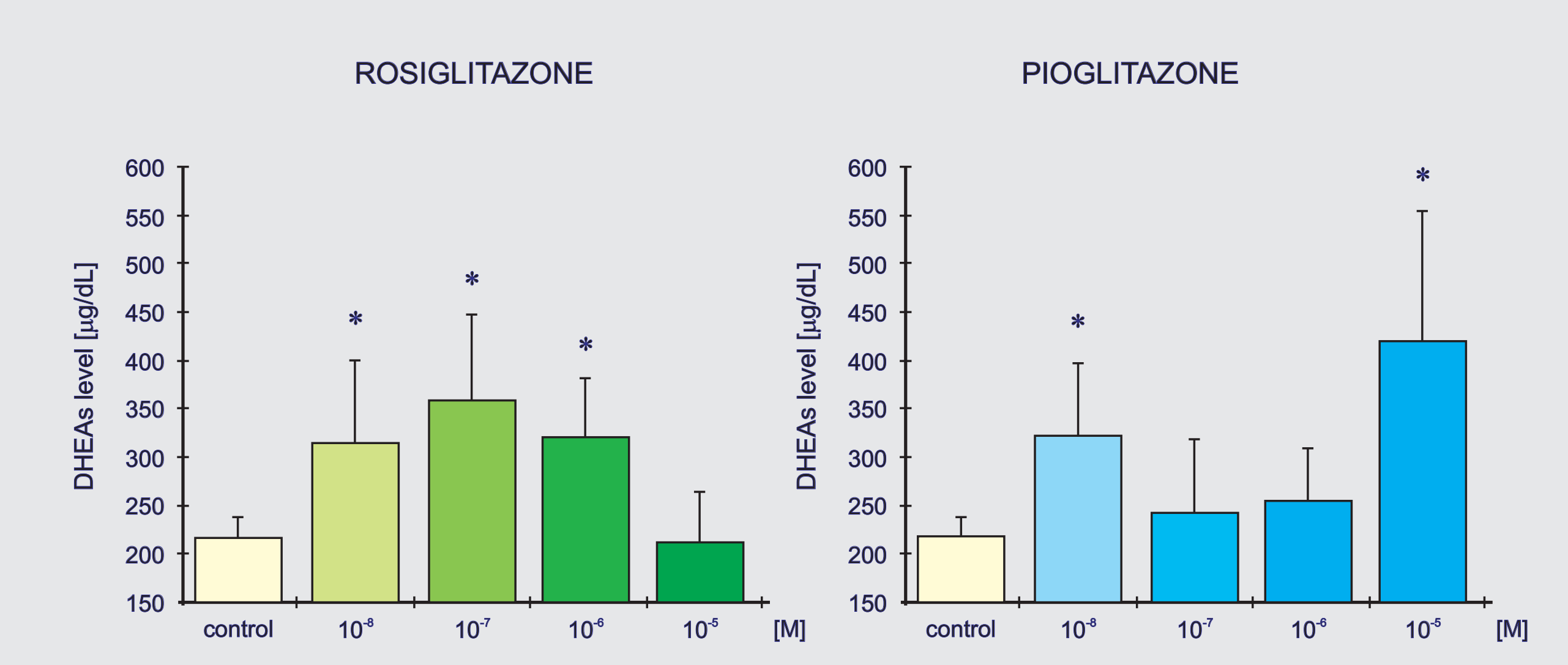

Fig. 1.

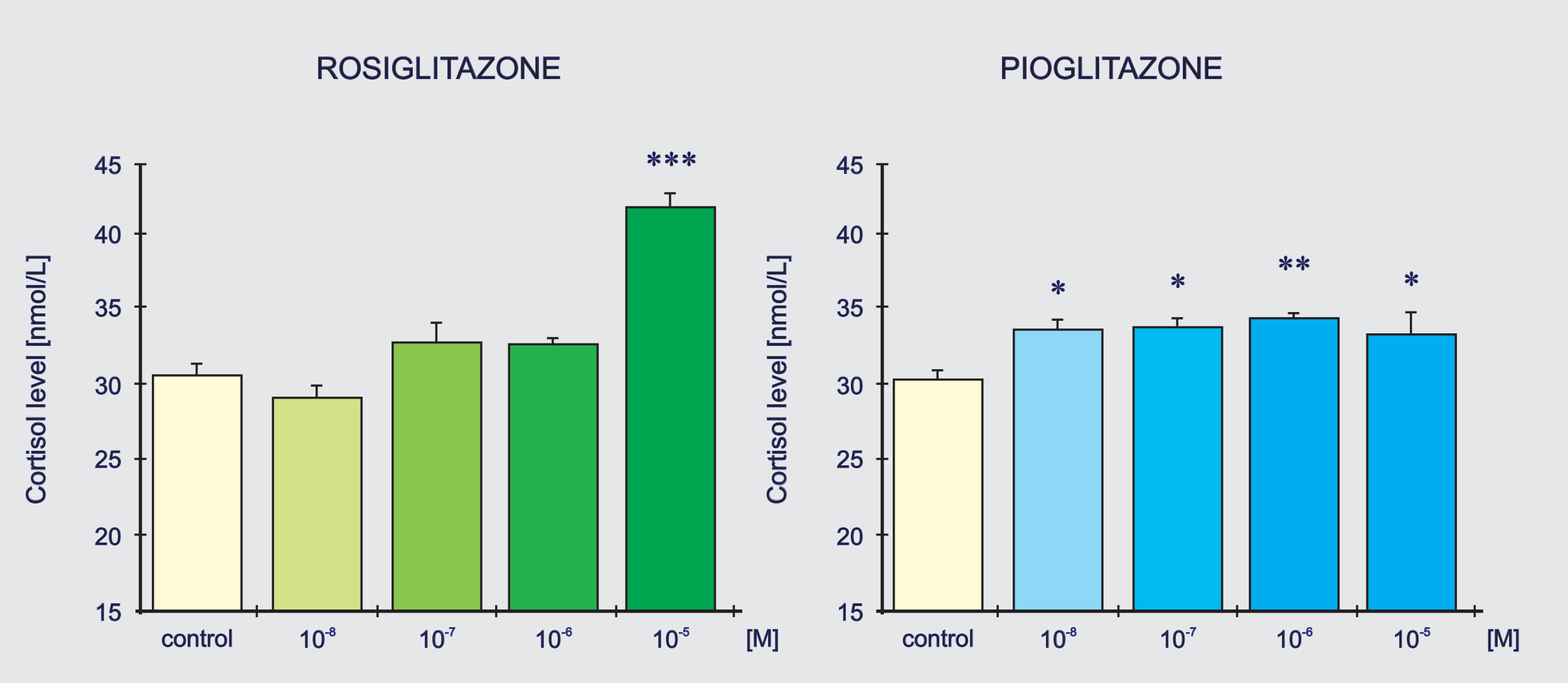

Fig. 2.

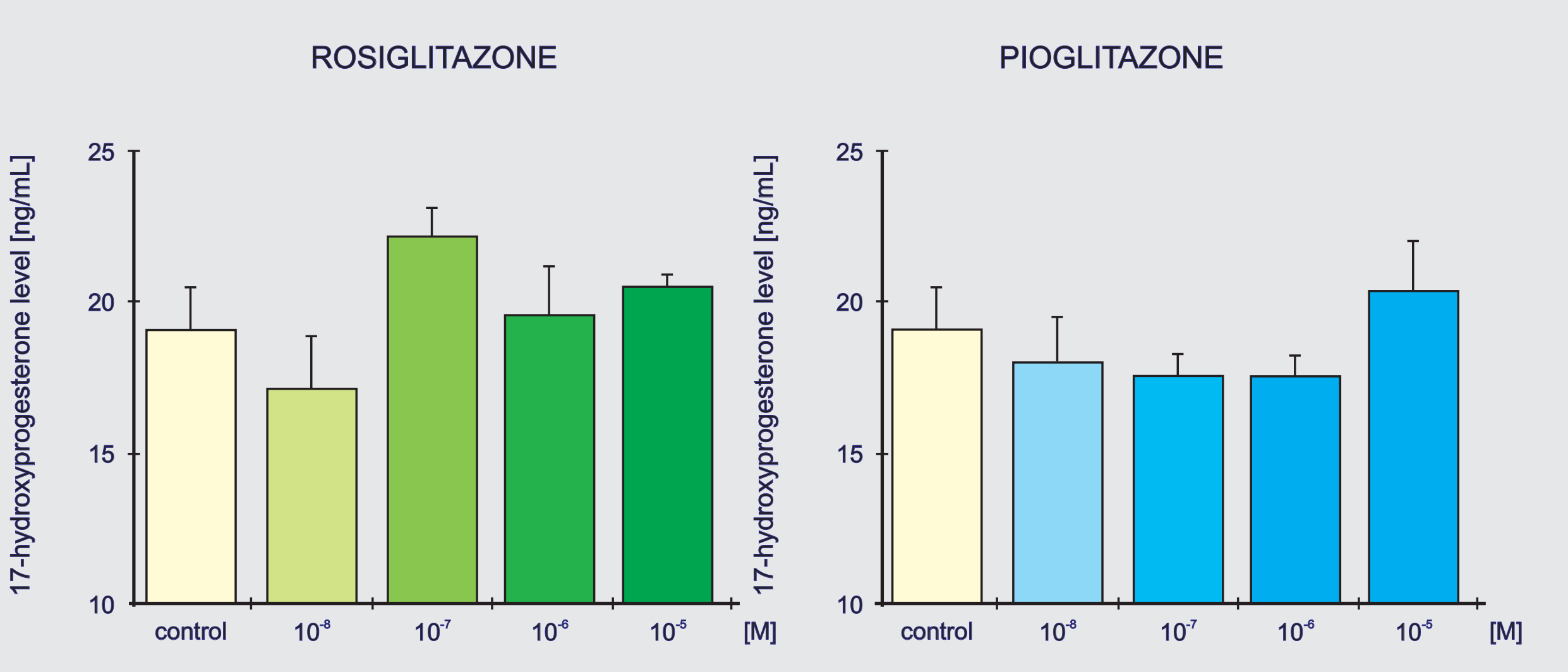

Fig. 3.

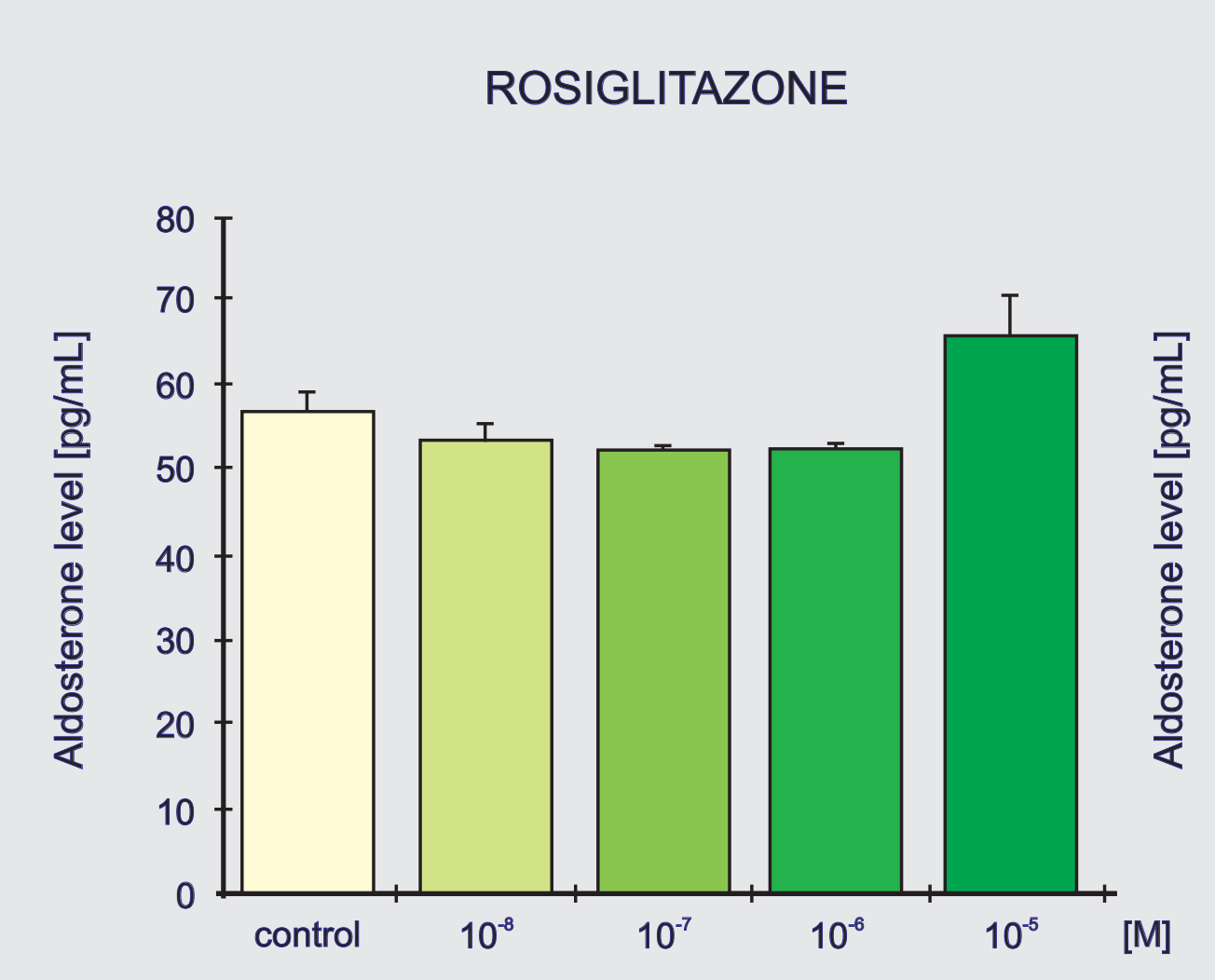

Fig. 4

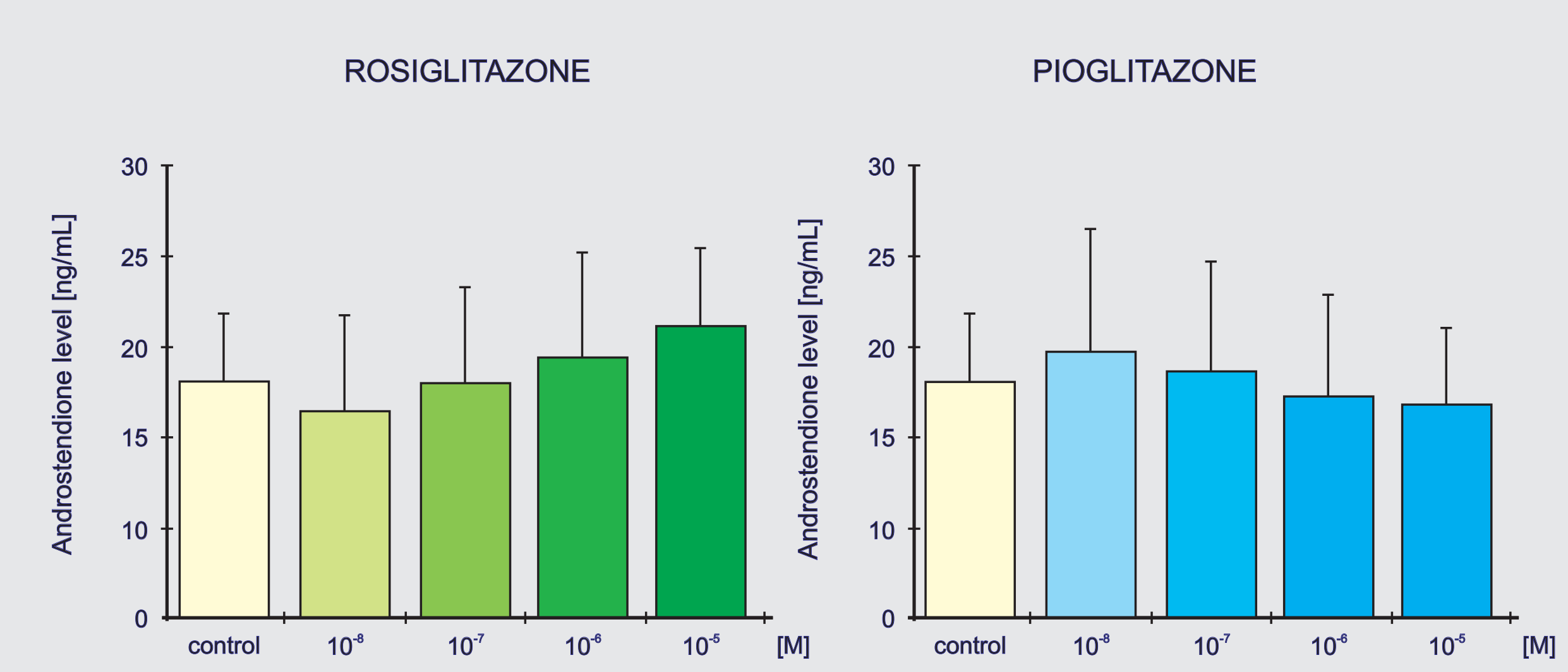

Fig. 5.

Fig. 1-5. Effect of rosiglitazone and pioglitazone on the hormones secretion by cells in H295R line.

\section{CONCLUSION:}

Agonists of PPARy, despite the inhibitory effect on the growth of adrenocortical carcinoma, do not reduce the hormonal activity of cancer cells. So it can be presumed that oncostatic action of TZDs and their influence on the synthesis of adrenal hormones are unrelated and depend on a different intracellular mechanism. 